\title{
Contaminación visual-ambiental de los soportes de las antenas de telecomunicaciones en el paisaje urbano
}

\section{Visual-environmental pollution of telecommunication antenna supports in the urban landscape}

\author{
DOI: 10.17981/mod.arq.cuc.26.1.2021.05
}

Artículo. Fecha de Recepción: 02/08/2020. Fecha de Aceptación: 27/11/2020.

\author{
Emerson Martínez Palacios (D) \\ Universidad de la Costa. Barranquilla (Colombia) \\ emartine62@cuc.edu.co
}

Para citar este artículo:

Martínez, E. (2021). Contaminación visual - ambiental de los soportes de las antenas de telecomunicaciones en el paisaje urbano. $M O D U L O$ ARQUITECTURA CUC, 26, pp. 113-136, 2021. DOI: http://doi.org/10.17981/mod.arq.cuc.26.1.2021.05

\section{Resumen}

El presente artículo tiene como centro la cuestión de la contaminación visual y ambiental causada por los soportes de las antenas de telecomunicaciones que se encuentran ubicadas dentro de los barrios o sectores urbanos consolidados, impactando en la formación de percepciones de abandono urbano y de inseguridad por parte de la ciudadanía; para ello se realizará un análisis de los diferentes impactos y de la percepción de seguridad que causan el establecimiento de los soportes de las antenas en el contexto urbano, determinando sus problemáticas urbanas. Posteriormente se abordará la discusión acerca de las actuales soluciones dadas para dichos soportes de las antenas desde aspectos visuales (mimetización) para finalmente concluir con aspectos proyectuales de diseño y gestión del espacio requerido para la instalación de estas antenas de telecomunicaciones en pro de la construcción de identidad urbana y no del deterioro del paisaje urbano.

Palabras claves: Contaminación visual; Percepción ambiental; Paisaje urbano; Antenas urbanas; Telecomunicaciones; Infraestructura urbana; Diseño urbano

\begin{abstract}
This article focuses on the issue of visual and environmental pollution caused by the supports of telecommunications antennas that are located within neighborhoods or consolidated urban sectors, impacting on the formation of perceptions of urban abandonment and insecurity on the part of citizenship; For this, an analysis will be carried out of the different impacts and the perception of security caused by the establishment of the antenna supports in the urban context, determining its urban problems. Subsequently, the discussion about the current solutions given for said antenna supports will be addressed from visual aspects (mimicry) to finally conclude with project aspects of design and management of the space required for the installation of these telecommunications antennas in favor of the construction of urban identity and not the deterioration of the urban landscape. Keywords: Visual pollution; Environmental perception; Urban landscape; Urban antennas; Telecommunications; Urban infrastructure; Urban design
\end{abstract}




\section{INTRODUCCIÓN}

La importancia que tienen las telecomunicaciones para el desarrollo y funcionamiento de la ciudad son incuestionables ya que ofrece beneficios de índole social, económica, productiva y cultural para con sus ciudadanos. No obstante, la red de telecomunicaciones en la ciudad requiere de la instalación de antenas cercanas y equidistantes en la ciudad para transmitir las comunicaciones necesarias, lo cual genera situaciones entrópicas con los barrios e impactos al medio ambiente y el paisaje urbano.

La normatividad colombiana contempla tres consideraciones técnicas para el despliegue de infraestructura de telecomunicaciones, las cuales están dirigidas a identificar las principales soluciones que se pueden aplicar para minimizar los efectos de las instalaciones de infraestructuras de las redes móviles al integrarse al entorno urbano que las rodea, las cuales están consignadas en el Código de Buenas Prácticas para el Despliegue de Redes de Telecomunicaciones expedido por la Comisión de Regulación de Comunicaciones (CRC) (Bustamante, Farías, Botero y Romero, 2000).

Haciendo especial énfasis en una de ellas, la cual se denomina mimetización, se establece como una estrategia para cuidar el medio ambiente y el patrimonio dependiendo del contexto y lugar en donde se decida instalar con el fin de reducir el impacto visual de los elementos a ubicar (Romero, Quintero, Cortes y Botero, 2016). Con lo anterior se busca que dicha integración confluya con el entorno del barrio, ya sea de manera natural emulando los soportes de las antenas como árboles de gran altura tales como las palmeras o mediante algún tipo de cubierta.

\section{Desarrollo}

\section{Caracterización de los lotes \\ para la infraestructura de la \\ red de telecomunicaciones en un barrio}

La infraestructura de telecomunicaciones que encontramos en un barrio, por lo general, ocupa un lote de dimensiones cercanas a los 80 y $120 \mathrm{~m}^{2}$, en el que dicho espacio es cerrado visual y físicamente con un acceso a personal de mantenimiento. En él se emplaza un cubículo para los equipos RF (Radiofrecuencia) y los demás equipos auxiliares. Sumados a estos se encuentran las torres o soportes para las antenas de telecomunicaciones que pueden llegar a ser de 20 a 40 metros de altura en las que en su parte superior reposan las antenas. Este lote debe cumplir con lo establecido en el Decreto 1077 (2015, art. 2.2.6.1.1.1):

Licencia urbanística. Para adelantar obras de construcción, ampliación, modificación, adecuación, reforzamiento estructural, restauración, reconstrucción, cerramiento y demolición de edificaciones, y de urbanización, parcelación, loteo o subdivisión de predios localizados en terrenos urbanos, de expansión urbana y rurales, se requiere de manera previa a su ejecución la obtención de la licencia urbanística correspondiente. Igualmente se requerirá licencia para la ocupación del espacio público con cualquier clase de amueblamiento o para la intervención del mismo salvo que la ocupación u obra se ejecute en cumplimiento de las funciones de las entidades públicas competentes (art. 2.2.6.1.1.1). 
La caracterización de estos lotes, tal como se muestra en la Figura 1, poseen muros cerrados sin permeabilidad visual que en el mejor de los casos cuenta con un acabado similar a las fachadas laterales, sin embargo, son carentes del intercambio visual y físico, que se denota en la morfología de las fachadas laterales al lote, y que se debe dar entre el espacio público y el privado. Los edificios de una calle equipada para superar la prueba de los desconocidos y, al mismo tiempo, procurar seguridad a vecinos y desconocidos, han de estar orientados de cara a la calle. No deben dar su espalda ni sus muros a la calle dejándola así ciega (Jacobs, 2011).

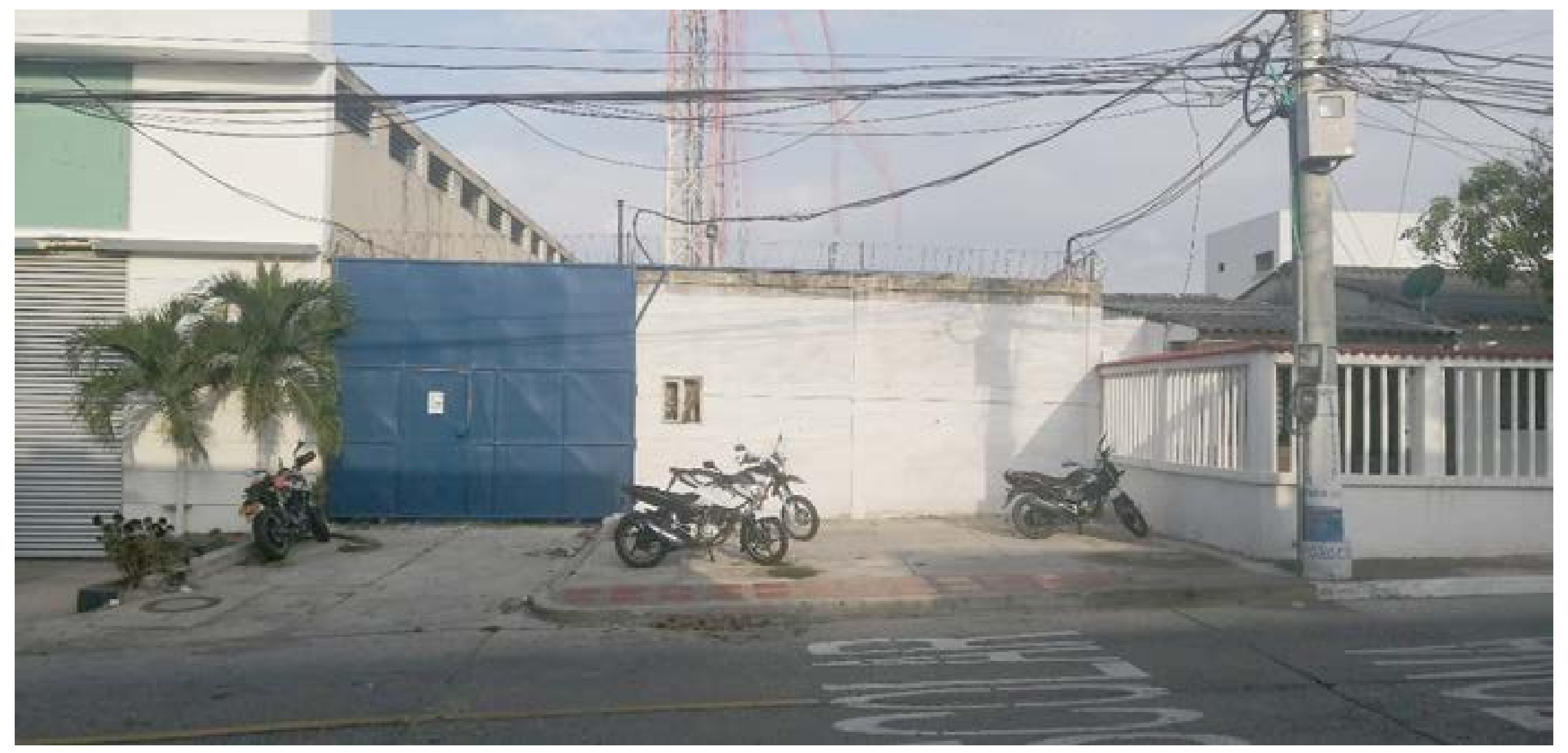

Figura 1. Lote cerrado para la instalación de infraestructura de telecomunicaciones.

Fuente: Archivo personal. 
Dicha configuración de fachada ciega relata la ausencia del uso ciudadano al interior del lote, lo cual crea connotación de abandono y por ende aumento de la percepción de inseguridad al exterior de él.

Necesidades del despliegue de redes de telecomunicaciones en las ciudades según tipo de zona

Dependiendo del tipo de zona en el que se vaya a realizar el despliegue de la red de antenas para las telecomunicaciones, se da la cantidad de soportes de antenas requeridos o necesarios para que los usuarios puedan hacer uso de esta red de manera eficaz.

El concepto de redes móviles está basado en subdividir áreas geográficas relativamente grandes en secciones pequeñas llamadas celdas o células; cada celda corresponde al área geográfica cubierta por una antena o por un sector de la estación base a la cual pertenece dicha antena. Estas áreas geográficas, pueden variar entre metros y kilómetros. Así mismo, de acuerdo con la cantidad de usuarios que deban ser atendidos, en determinadas celdas se requerirá mayor cantidad de antenas para satisfacer la demanda del servicio (Bustamante et al., 2020).

Lo anterior muestra que técnicamente no es apropiado definir distancias fijas entre antenas, dado que su ubicación debe atender a las necesidades específicas de la zona a cubrir tales como cantidad de usuarios que se conectan de manera simultánea y obstáculos presentes en el ambiente. Las antenas al hacer uso del espectro requieren ser instaladas en espacios abiertos, y por ende no resulta viable su ubicación de manera subterránea. También es de mencionar que entre mayor sea la población en una zona, dadas las condiciones técnicas de cobertura y capacidad de cada estación base, habrá una necesidad de instalar mayor cantidad de antenas para atender a todos los usuarios y mantener la continuidad y calidad del servicio (Bustamante et al., 2020).

De acuerdo con la Figura 2, se denota la cantidad de antenas necesarias teniendo en cuenta las diferentes zonas urbanas: periférica, central y residencial, y la concentración de usuarios contenida en ellas. De esta manera, a mayor usuarios y obstáculos físicos (tales como las edificaciones, colinas, etc) mayor número de antenas y por ende soportes para las mismas, las cuales deben disponerse en lotes, fachadas y terrazas.

A continuación, veremos los diferentes tipos de soporte para antenas de telecomunicaciones y sus instalaciones al interior de la ciudad y sus barrios, con lo cual se inicia la observación del impacto visual y afectación que estas generan en el paisaje urbano. 


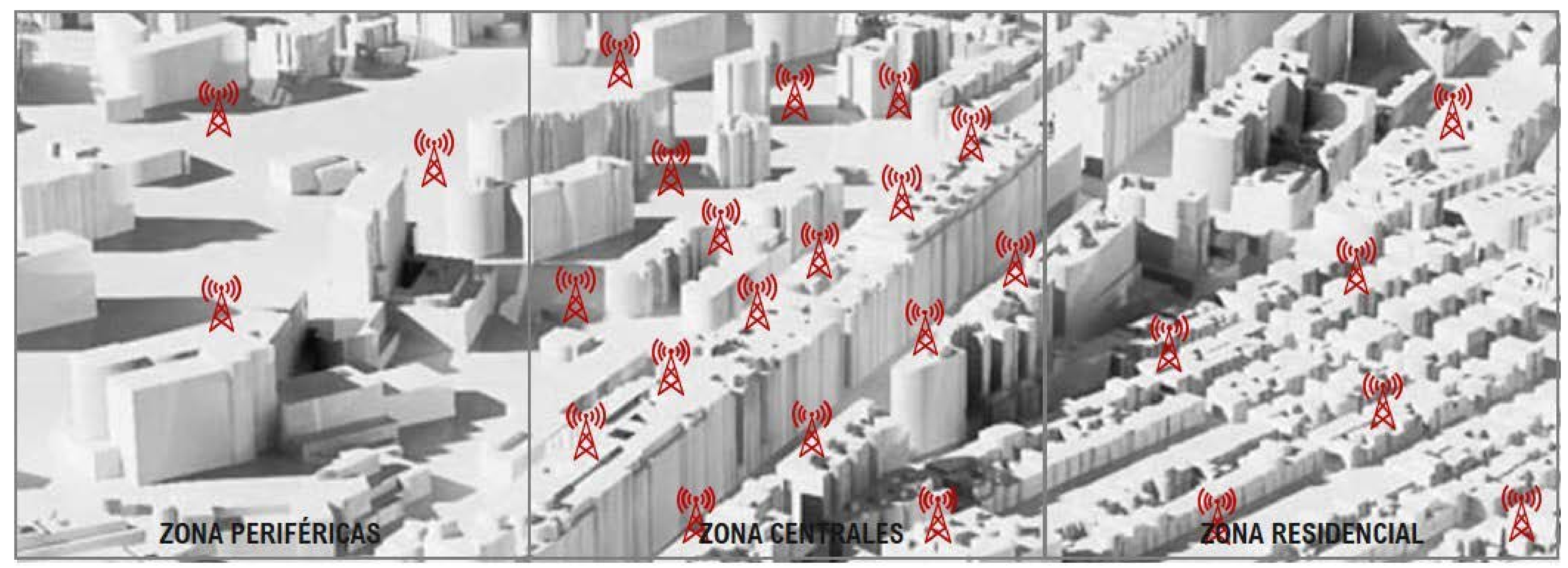


Tipos de estructuras de soporte para antenas

Una estructura soporte para antena es un objeto compuesto por una estructura metálica realizada por con perfiles y/o tubos de acero en donde se fijan las antenas de VHF, UHF, Internet, microondas, parabólicas y de radio, mediante accesorios determinados a, mástiles, paredes, postes o torres de telecomunicaciones. Se encuentran de los siguientes tipos (Figura 3):

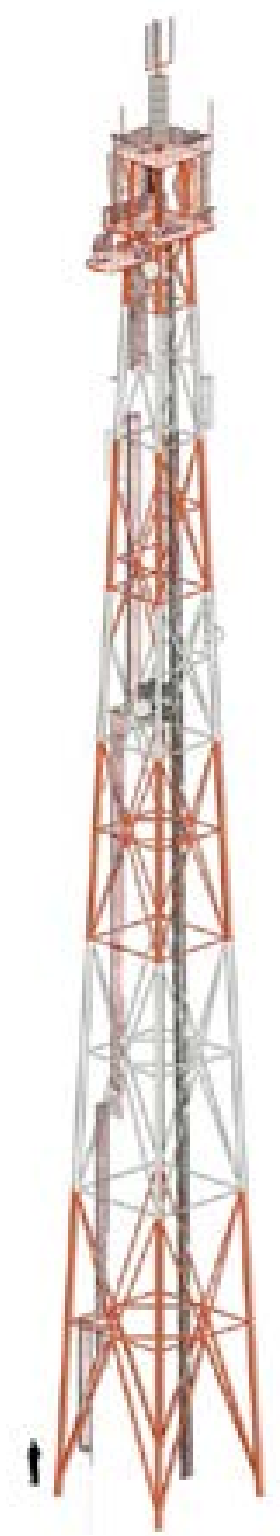

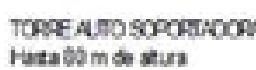

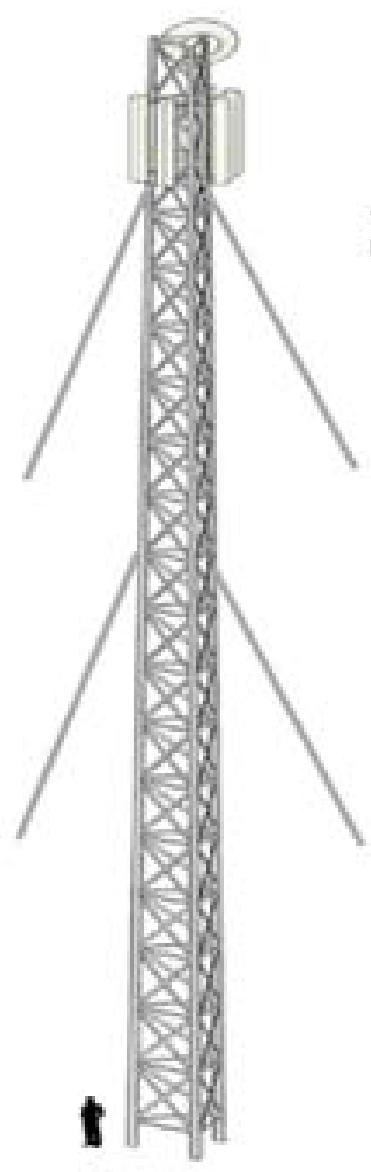

toreracora Heta 60 inding ats

Figura 3. Tipos de estructuras de soporte.

Fuente: Bustamante et al. (2020). 
Con respecto a las anteriores, se tendrá en cuenta para la referenciación de este estudio su afectación en el paisaje urbano las estructuras de soporte para antenas de tipo Torre Aut soportadora y Monopolo, siendo estas las más comunes que se encuentran en los barrios o sectores urbanos residenciales.

Afectaciones del paisaje urbano por las estructuras de soporte para antenas

Se reconoce el paisaje urbano como el conjunto de elementos que componen la visualización de la interacción entre el espacio público y el privado. Cada uno de estos ele se relacionan desde aspectos funcionales y estéticos, lo cual refiere a que la morfología del paisaje urbano se estructura de acuerdo con estas relacione de manera consecuente o no.

Dado que el paisaje urbano es la expresión de todo lo que es posible percibir en los espacio públicos de la ciudad, el análisis de los atributo urbanos permite identificar patrones, secuencias y unidades del paisaje urbano que conducen a establecer criterios de diseño en término de calidad visual (Briceño-Ávila, 2018). Esta calidad visual llega a afectarse cuando alguno

Figura 4. Afectaciones del paisaje urbano. Fuente: Archivo personal.

de los elementos no se relaciona desde aspectos morfológicos, ni teniendo en cuenta la estética del paisaje urbano. En este caso, las estructura de soporte para antenas de tipo Torre Auto so-

portadora y Monopolo, con sus alturas hasta de la percepción del paisaje urbano, sumado a ello, las características constructivas obedecen a una estética que promulga aspectos industriales, que van desde el color hasta la configuración formal dada por estructuras triangulares y tubos en secuencia diametral.

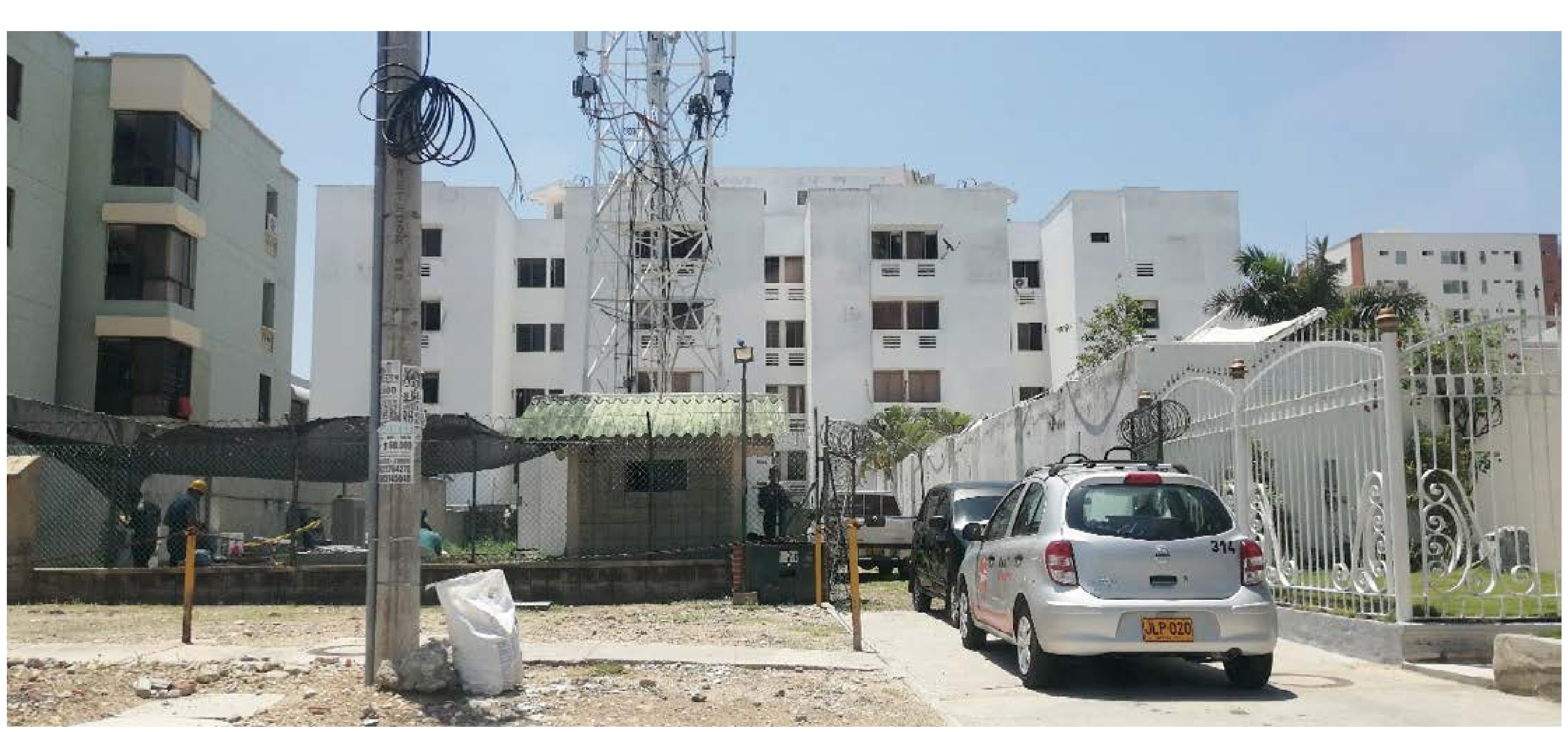


Haciendo énfasis en los efectos sobre el paisaje urbano que causan la existencia de los soportes para antenas instalados en los barrios, puede denotarse en la Figura 4, afectaciones que se contemplan desde el plano horizontal (la acera, su configuración formal, materiales y acabados) su confo a ques un lote cuyo uso de siblos) debido a pu sue no pertenece a las funcions haciendo que sus condicios de habitabilida se vean reflejach en sure nal. De igual manera sucede con la morfología del antejardin, el cual las residencias laterales han desarrollado con elementos de cerramiento acordes en têrmi cos de color y perímetro del área, generando una diferenciación tácita del inexistente cerramiento frente al lote dispuesto para la Torre Auto soportadora de las antenas.

Habrá que tener en cuenta que instalaciones como estas se repiten con frecuencia (Figura 2), multiplicando los efectos visuales y las posibilidades de contaminación visual y de percepción de inseguridad, alterando el paisaje urbano en los barrios.

- Contaminación visual

La contaminación visual abarca un amplio número de elementos que la producen, entre ellos todos aquellos que alteran el paisaje urbano, tal como lo expone Rozadas (2006) en su definición "abuso de ciertos elementos -no arquitectónicos-, que alteran la estética, la imagen del paisaje rural o urbano" (p. 1).
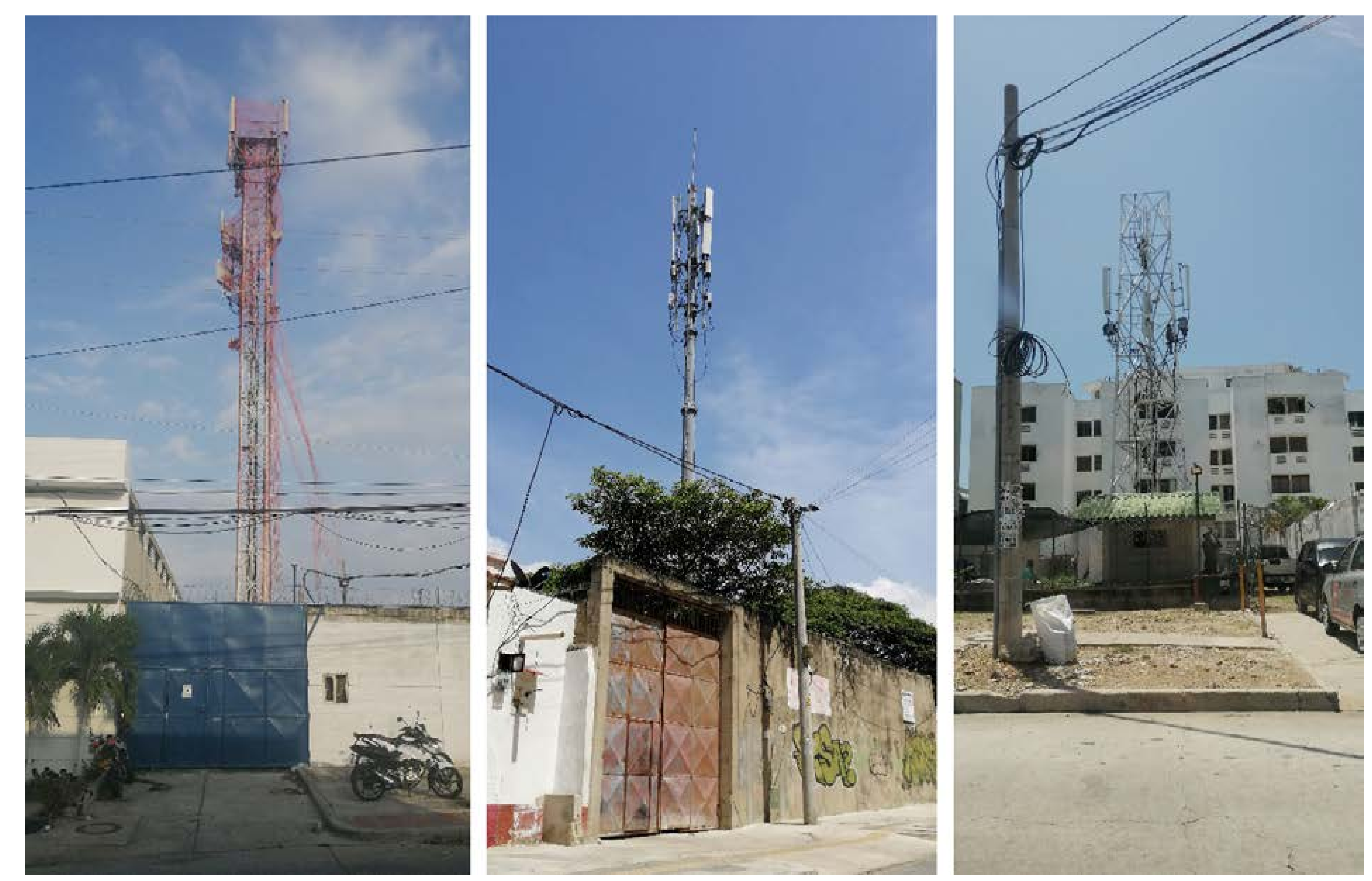
Si bien es cierto que la estética arquitectónica de un barrio es perteneciente al ritmo de lo edificado, a los colores, morfologí y funcones establecidas en las fachadas, y estas a su vez en el lleno visual que permite una continuidad de la calle, la prescia de los vacios, la atura de

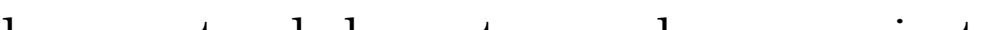
conformando fachas ciegas, crean una desconformando fachadas ciegas, crean una desde la de la configuración arquitectónica de un barrio residencial generando patrones contaminantes desde lo visual. Por lo anterior, se considera que el establecimiento de las estructuras soportes de las antenas en los barrios, alteran la estética arquitectónica y suman aspectos de desequilibrio y contaminación del equilibr urbano en las zonas residenciales, consolidando percepciones y consecuencias visuales que deterioran el paisaje urbano. La suma de esta alteraciones genera saturaciones visuales 1 cual no solo fractura la lectura del paisaje urbano, sino también connota percepcione contraproducentes para la identidad urbana de los barrios.

El manejo abusivo de los mismos por parte del hombre y la falta de planeación urbana, generan en la percepción del ciudadano una sobre estimulación visual excesiva, agresiva e indiscriminada, que no solo termina con deteriorar la imagen de la ciudad, sino también, apropiación ciudadana (Sierra y Guevara, 2016)
Dicha apropiación se denota desde la manera como la ciudadanía percibe el espacio urbano circundante a los lotes que contienen los soportes para las antenas de telecomunicaciones, por lo cual será importante ampliar el concepto de percepción ambiental.

- Percepción ambiental

Con respecto a la percepción ambiental, la visualización es una de las actividades preponderantes, ya que con ella se logra entender el espacio observado y comprender el mensaje que se da in situ mediante la composición de los elementos urbanos. Así las cosas, la percepción ambiental abarca dos factores claves: El observador que es el Ciudadano y el espacio observado, el cual está compuesto por diferentes elementos que denotan una realidad.

Realizando un análisis sobre la Figura 6 se denotan elementos visuales y físicos que alteran la morfología del barrio, fisicos que grafitis de expresión urbano-artística y otros de expresión vandálica, pintura en mal ostado in cercen funcion uranos de apertura y de relación

Figura 6. Percepción ambiental urbana de soportes de antenas y su espacio circundante. Fuente: Archivo personal.

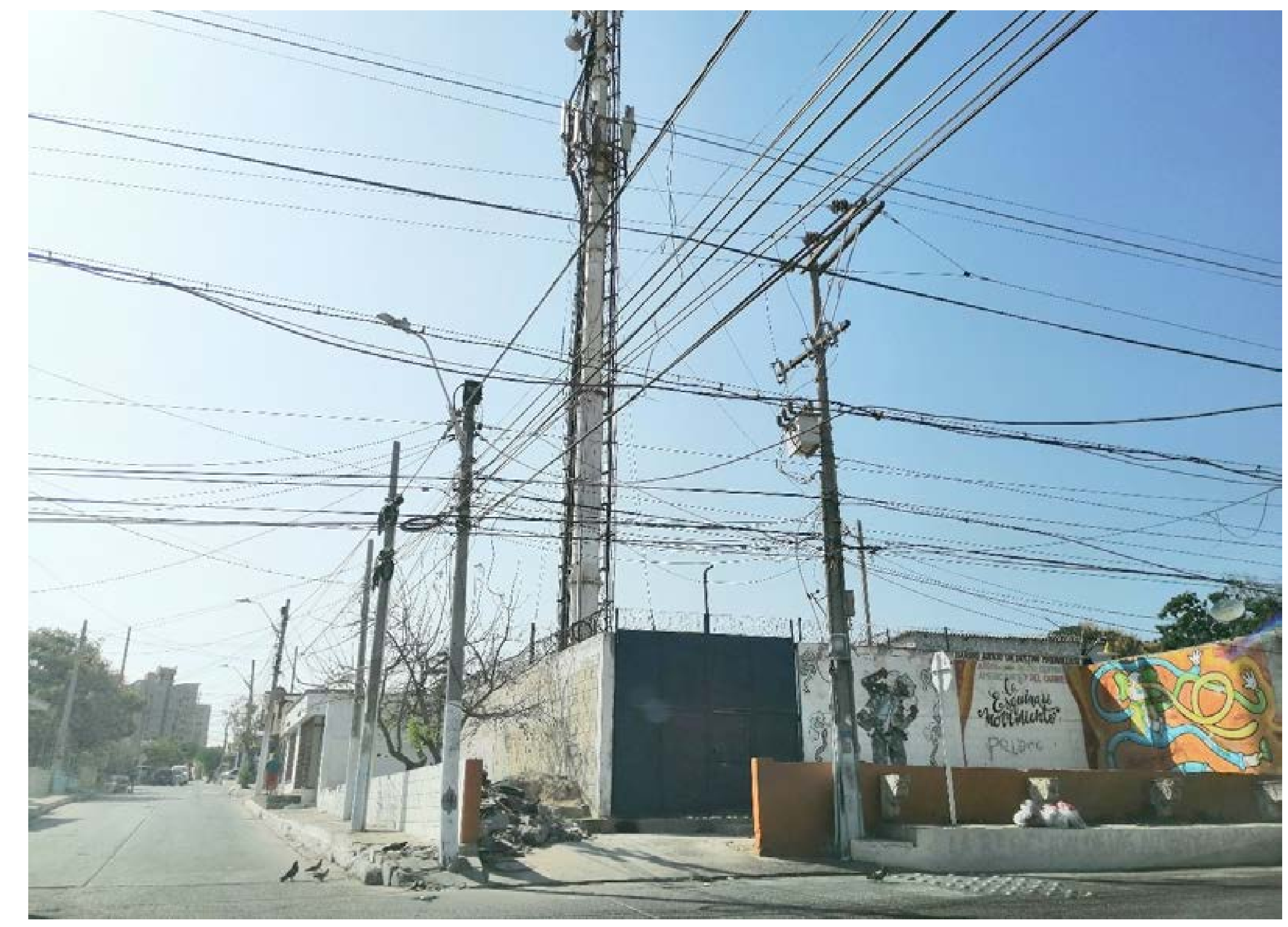


entre lo privado y lo público, cercado superio del espacio cerrado con puas en espiral (connotando agresividad), espacios cedentes en donde se da la acumulación de escombros, basuras acumuladas frente a la fachada (siendo este una aspecto recurrente a las instalaciones de la infraestructura de telecomunicaciones por falt de habitabilidad del espacio cerrado), y finalmente, el mayor elemento de dicha alteración: la estructura soporte de las antenas (en este caso el tipo Monopolo de hasta $40 \mathrm{~m}$ de altura en un barrio en donde sus residencias, en su mayoría, son de una planta).

Detallando en el sentido de la percepción am biental del espacio circundante a los espacios destinados para las antenas de telecomunicaciones, se tiene una caracterización recurrente en los que se dan las siguientes expresiones que redundan en aspectos de percepción de ins qu ridad: Espacio público deteriorado, no lugare que los vecinos utilizan pera arrojar escombes que los vecinos ufitis vandálicos, car escombros y/o basu ece giezas de in pintura desación de basurasen la fach o
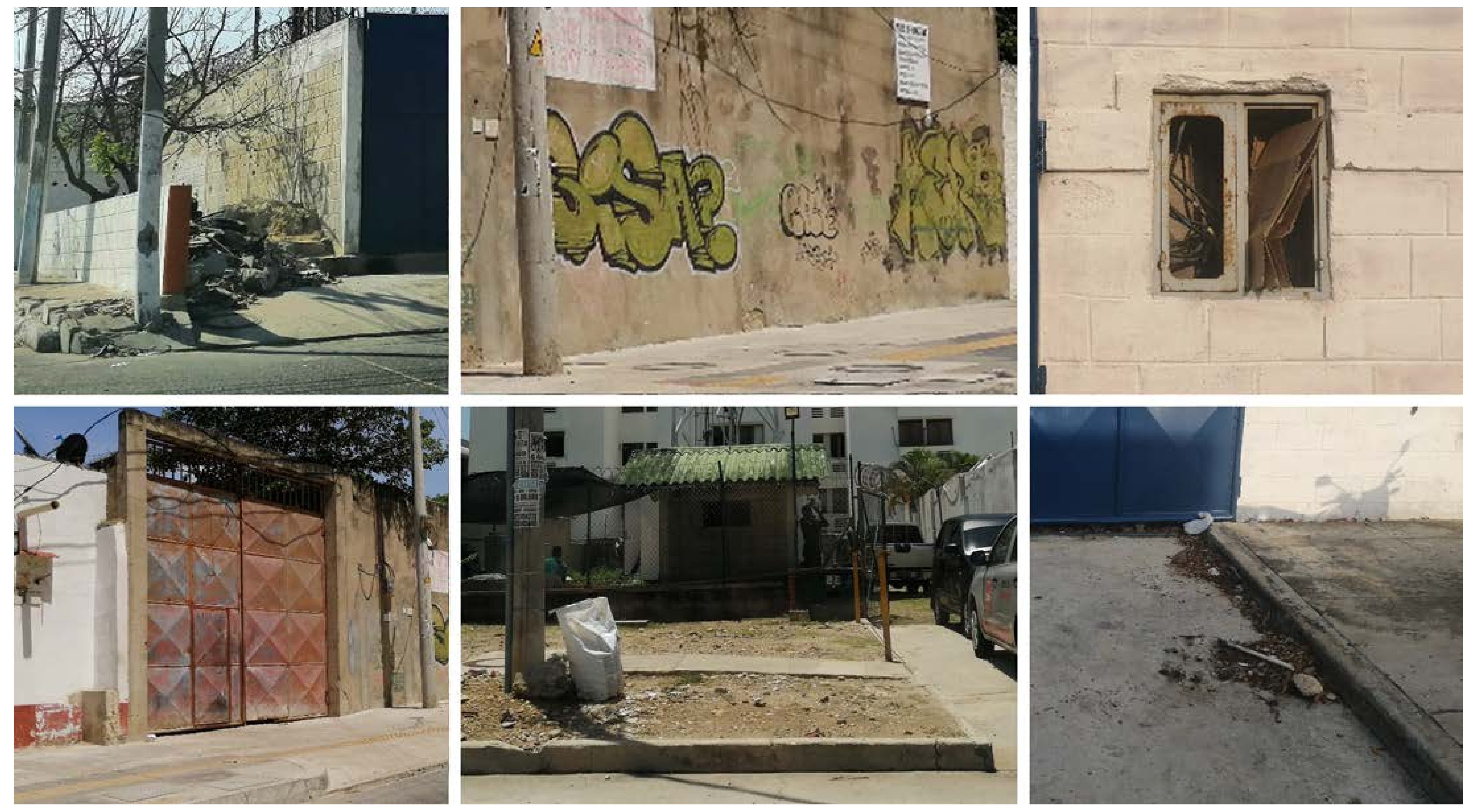
Cada uno de los elementos anteriores produce estímulos adversos que llegan a proyectarse en la actitud de los ciudadanos hacia su propio espacio público y, por ende, al paisaje urbano del barrio, generando apropiaciones del entorno urbano que acentúan la contaminación visual ya creada.

Esta situación genera grandes inconvenientes urbanos, puesto que, sumado al deterioro físico causado por la contaminación visual, la crisis ciudadana de desaprobación y desarraigo por los lugares contaminados visualmente, que no brindan comodidad y bienestar físico, generan una desconexión con la ciudad y una falta de responsabilidad con la imagen de la misma. Un espacio urbano poco legible es difícilmente legitimizable, por cuanto dificulta la identificación del habitante con su hábitat, del ciudadano con su ciudad; es un espacio deteriorado no solo estéticamente sino, socialmente. Es un espacio deshumanizado (Sierra y Guevara, 2016).

\section{Metodología}

Para el desarrollo del presente artículo se llevó a cabo la observación directa mediante la elaboración de un registro fotográfico de seis (6) diferentes y recurrentes elementos contaminantes visuales dispuestos en la observación de doce (12) instalaciones de la infraestructura de telecomunicaciones en sectores residenciales y/o barrios. Con el fin de detallar los aspectos conceptuales de la observación directa, se aplicó el método deductivo simple, con el que se estructuró un marco referencial en el que se abordó la caracterización del despliegue de redes de telecomunicaciones y sus tipos de soporte. Posteriormente se introdujo un marco teórico haciendo referencia los factores principales que consolidaron las afectaciones del paisaje urbano por las estructuras de soporte para antenas, buscando proyectar una problemática urbano ambiental. Finalmente, se consolidan como resultados del presente estudio, requerimientos objetuales para tener en cuenta a fin de proyectar infraestructuras de telecomunicaciones que dialoguen con la morfología y funciones propias del espacio público y del barrio.

\section{Resultados}

Con el fin de que la proyección de los espacios destinados para la infraestructura de telecomunicaciones sea acorde a los aspectos morfológicos de los barrios y articulados con los barrios desde caracteres estéticos entre el diseño visual-formal y funcional, se plantea el desarrollo de requerimientos de diseño para tenerlos en cuenta en una nueva posible concepción de espacio, que se articule con las condiciones urbanas de los barrios y las necesidades de espacio público que, en términos generales, se desarrolla en los barrios. Con lo anterior se busca que dichos espacios se conciban y proyecten de manera abierta y, en consecuencia, bajo caracterizaciones de funciones y usos tal como los que posee el espacio público.

Realizando la observación sobre los espacios destinados en los barrios para la instalación de la infraestructura de los servicios públicos tales como el acueducto, la energía y/o el gas, estas se dan sobre la superficie del espacio público y debajo de su plano horizontal (suelo), denotando 
que el espacio público es el soporte de dichas infraestructuras. Ahora bien, haciendo un símil con lo anterior, la infraestructura necesaria para las telecomunicaciones que se despliegan en los barrios, al tener la misma caracterización servicio público, deben consecuentemente formalizarse desde los parámetros y caracterización que se establece para la proyección de los espacios públicos; de esta manera se plantea como resultado del presente estudio un listado de requerimientos a tener en cuenta para la proyección de los espacios destinados para el despliegue de la infraestructura de telecomunicaciones en los barrios.

Requerimientos objetuales para estructuras de soporte para antenas y su espacio circundante

Los requerimientos de diseño son variables que deben cumplir una solución cuantitativa y cualitativa, siendo fijadas previamente por una decisión, por la naturaleza y por requisitos legales, o por cualquier otra disposición que tenga que cumplir el solucionador del problema. Variables que limitan las alternativas del solucionador de productos. Observación: el termino requerimiento es sinónimo de restricción, especificación, consideración variable (Rodríguez, 1983).

Aunque se reconoce que los parámetros para el despliegue de infraestructuras de telecomunicaciones ya se encuentran establecidos, se aportan los siguientes requerimientos a fin de proyectar soluciones hacia las situaciones de contaminación visual, percepción ambiental y articulación urbana:
- $\quad$ Requerimientos de uso:

El espacio circundante (lote) a la estructura soporte de las antenas debe posibilitar su uso por parte de la ciudadanía en donde sus vecinos obtengan el disfrute de dicho espacio con consideraciones de uso especiales desde los aspectos tecnológicos de las telecomunicaciones, es decir, la obtención de actividades interactivas mediadas por las tecnologías de conectividad. El fin de proyectar este uso es que se consoliden nuevas maneras de interacción entre ciudadanos mediadas por las tecnologías de la información y la comunicación.

\section{- Requerimientos de función:}

Dando alcance a las posibilidades de uso del espacio (lote) desde los parámetros de espacio público, este ha de ser abierto y accesible, prestando funciones físicas de mediador de las interrelaciones de los ciudadanos en donde el intercambio y la comunicación se consolide como una función más sumada a las de las telecomunicaciones. Por lo anterior, debe ser un soporte de funciones urbano - ciudadanas tales como la contemplación, el descanso, la permanencia y reiterando: el intercambio social.

- $\quad$ Requerimientos formales $y$ de identidad urbana:

Aprovechando la altura de los soportes de las antenas de telecomunicaciones, estos pueden llegar a considerarse como referentes de ubicación y como "elementos de identidad" (Vidal, Salas, 
Viegas, Esparza y Padilla, 2012) de los barrios, o bien, como los denomina la teoría urbana: "hitos urbanos". Estos son puntos de referencia que deben ser impactantes al momento de visualizarlos (Lynch, 1960). De esta manera se tiene la oportunidad de concebir la estructura del soporte de las antenas más como un objeto de arte público y no sólo como una infraestructura física para soportar las telecomunicaciones de un barrio en una ciudad.

\section{- Requerimientos tecnológicos} y de interacción:

Aprovechando la tecnología de telecomunicación que se provee desde las antenas, se considera que gracias a ello estos espacios pueden llegar a ser configurados como zonas de interacción digital, mediante actividades lúdicas y procesos de comunicación digital abiertos a la ciudadanía, tal como se denotan en espacios públicos con conectividad en las ciudades contemporáneas. De esta manera se busca que se generen zonas de innovación cultural y creativas, lo cual posibilitan la transformación de imaginarios colectivos, fundamentando sus identidades y consolidando su imagen (Padilla et al., 2020).

- Requerimientos de articulación urbana:

Será importante que las funciones del espacio referido a la infraestructura de telecomunicaciones sean acordes a las necesidades y activi- dades sectoriales de la ciudad, buscando que se den oportunidades de significación urbana mediante las actividades que se pueden desarrollar sobre él, así como lugares de encuentro e innumerables formas de aprovechamiento, logrando adaptarse a la organización del barrio (Martínez, 2013). Así las cosas, el espacio en mención debe basarse en el desarrollo equilibrado de todos los determinantes y escalas de la ciudad y la arquitectura. Esto constituye un concepto alternativo e integrador del Espacio Público como unidad de vida urbana y ambiental y como escenario de la socialización (CabasGarcía, 2019).

\section{Discusión}

Para abordar la discusión del presente artículo se realiza desde el cuestionamiento: ¿es la mimetización una solución para la contaminación ambiental del paisaje urbano y para la percepción de inseguridad generada por el despliegue de los soportes de las antenas de telecomunicaciones?

En respuesta a dicho cuestionamiento se define cual es la caracterización de la mimetización y/o camuflaje de las estructuras de soporte para antenas, con relación a contextos urbanos en donde el despliegue de infraestructuras de telecomunicación son puntos de encuentro y de interacción formalizados mediante espacio público para la accesibilidad de los ciudadanos. 
Mimetización y camuflaje de las estructuras de soporte para antenas

Según la Real Academia de la Lengua Española (RAE) se define mimetizar como: "Adoptar la apariencia de los seres u objetos del entorno"; es decir, que lo que se pretende al mimetizar las estructuras y componentes que conforman las estaciones de telecomunicación inalámbrica, es minimizar y reducir al máximo posible el impacto negativo que pueden tener sobre el entorno próximo en donde se instala dicha estructura, integrándolas a su contexto más inmediato. Por otra parte, se define camuflar como: "Disimular dando a algo el aspecto de otra cosa" (RAE); es decir que lo que se busca con los procesos de camuflaje de este tipo de estructuras es que, si bien su existencia y presencia es necesaria para prestar el servicio, es muy importante que los operadores y empresas instaladoras armonicen dicha existencia con su entorno físico, para que de esta manera el impacto sobre el medio ambiente y los seres humanos sea mínimo (Alcaldía Mayor De Bogotá, 2011)

Figura 8. Mimetización y camuflaje de las estructuras Fuente: Google Maps.

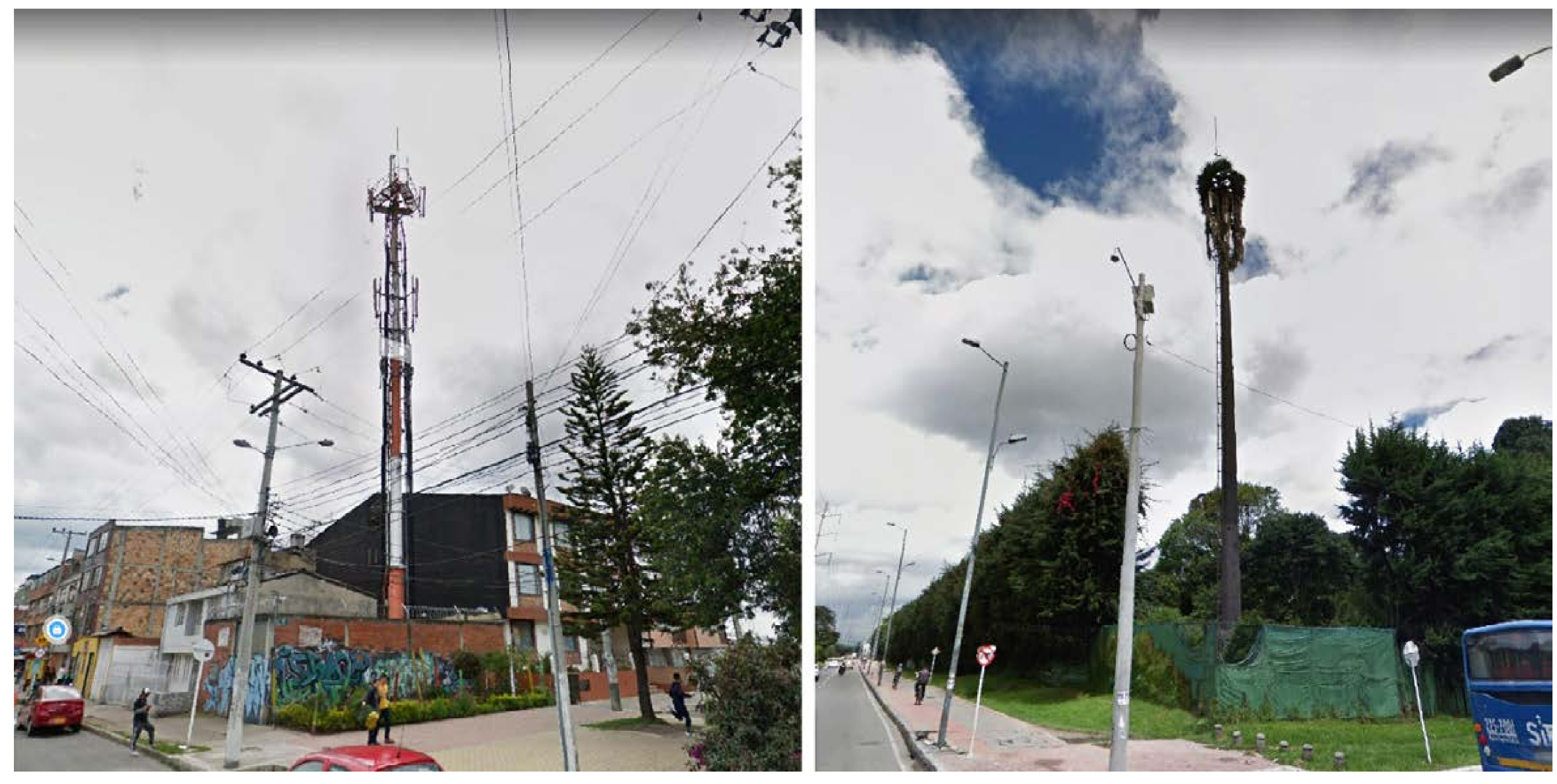



en el que se instala $\mathrm{y}$ cuando sea posible debe convertirse en un elemento decorativo del entorno urbano. De acuerdo con la autoridad de telecomunicaciones de Australia (Western Australian Planning Comission, 1928) la infraestructura de telecomunicaciones que es poco estética visualmente debe, en lo posible, ser instalada en zonas industriales, comerciales o rurales donde ese impacto se minimice. De lo contrario debe integrarse al entorno en función del diseño existente (Entel, 2012).

Por lo anterior, la mimetización busca obtener un proceso de asimilación (en su mayoría) a un contexto vegetal, no obstante, este esfuerzo por parecerse como un elemento natural se denota infructuoso, ya que la configuración ele cio y la estructura de soporte de las antenas de telecomunicaciones sigue teniendo los aspectos tubulares o estructurales propios desde los tipos de soportes (Figura 8), y sumado a lo anterior, de soportes ( en los barrios dicha mimetización no ocurre, sino que su estructura es la convencional, tanto en la configuración del espacio como en el soporte de las antenas (Figura 8).

En consecuencia, la mimetización de las antenas no establece un equilibi fionte a la alteración que generan en el paisaje urben ni tampoco define la sollcín a la contaminacion visul y la defne la son a gen atura, la moló de su fach y los as a su altura, la morfología de su fachada y los aspeca un contexto natural sin lograrlo.
El espacio para el despliegue de la infraestructura de telecomunicaciones como un lugar público de interacción y referente urbano

De acuerdo con los requerimientos formulados anteriormente, y retomando la concepción de la apertura del espacio hacia la formalización pública y la inclusión de factores de diseño formal estéticos guiados hacia concebir la estructura del soporte de las antenas más como un objeto de arte público, se logra consolidar una solución para la actual contaminación visual del paisaje urbano y mejorar la percepción de inseguridad.

Esto lo podemos ver en la gestión del espacio y de la infraestructura de telecomunicacione realizada en la Ciudad de Barcelona, con motivo de los juegos olímpicos de Barcelona de 1992 (Figura 9). Guardando las proporciones de espacio y altura, este lugar consolida y denota las posibilidades que se tienen actualmente para reformular las prácticas para el despliegue de redes de comunicaciones en los barrios de nuestras ciudades.

Figura 9. Torre de comunicacions de Montjuic Fuente: https://lommononswikimedia.org//wiki/File:Torre_de_comu-
nicacions_de_Montiuic1_-_Barcelona_(Catalunya).jpg 


\section{Conclusiones}

Ocasionalmente, resalta en el paisaje urbano, árboles, palmeras y elementos verdes con unas proporciones en altura y forma que superan la realidad natural. Para sorpresa y detallando la observación, resulta que no hacen parte de la naturaleza, ya que son antenas de telecomunicaciones.

La gestión de la red de telecomunicaciones en los barrios produce un alto impacto sobre el paisaje urbano y la percepción de seguridad de los barrios, debido a que la instalación de elementos físicos como torres auto soportadas, riendadas, monopolos y mástiles, presentan configuraciones formales que alteran dicho paisaje. Como resultado de esto, se han desarrollado estrategias tales como la mimetización, la cual busca que la infraestructura se integre al entorno en el que se instala, sin lograr su cometido.

Podemos tomar lo anterior como una causa para repensar las posibles antenas de la ciudad como hitos urbanos del barrio, en donde la oferta profesional y las posibilidades creativas del mercado pueden convenir para proyectar diseños, que hagan de la proyección de los espacios y soportes de las antenas urbanas de telecomunicaciones referentes visuales, haciendo expresa la capacidad que se tiene desde el diseño visual, industrial y arquitectónico, buscando obtener nuevos paisajes urbanos en los que estos elementos verticales y esbeltos, sean tratados como objetos de diseño, de arte público y de referencia de ubicación e identidad urbana y no que se asemejen pintorescamente a lo que nunca llegarán a ser: una especie vegetal.

\section{REFERENCIAS}

Alcaldía Mayor de Bogotá. (2011). Manual de Mimetización y Camuflaje de las estaciones de telecomunicaciones inalámbricas para el Distrito Capital. Bogotá, D.C.: Bogotá Humana. Disponible en https://oab. ambientebogota.gov.co/?post_type $=\mathrm{dlm}$ download\&p=3497

Cabas-García, M. (2019). Articulaciones urbanas, sistemas de relaciones espaciales y espacio público. [Online]. Disponible en http:// hdl.handle.net/11323/2467

Briceño-Ávila, M. (2018). Paisaje urbano y espacio público como expresión de la vida cotidiana. Revista de arquitectura, 20(2), 10-19. https:// doi.org/10.14718/RevArq.2018.20.2.1562

Bustamante, C., Farías, A., Botero, J. y Romero, F. (2020). Código de Buenas Prácticas para el despliegue de redes de comunicaciones. Bogotá, D.C.: Comisión de Regulación de Comunicaciones. Recuperado de https:// www.crcom.gov.co/uploads/images/files/ Buenas_Practicas_Despliegue_2020.pdf

Entel. (2012). Las antenas que Chile necesita. [Catálogo de integración urbana para estructuras de soporte de telefonia]. Santiago de Chile: Entel. Recuperado de https:// www.entel.cl/landing_antenas/mas_info/ entel_catalogoantenas.pdf

Jacobs, J. (2011). Muerte y Vida de las Grandes Ciudades Americanas. [2 ed.]. Navarra: Gráficas Lizarra.

Lynch, K. (1960). La imagen de la ciudad. Barcelona: Gustavo Gili. 
Martínez, E. (2013). El modelo Barcelona de espacio público y diseño urbano. El espacio público asociado a la infraestructura de movilidad rápida y rodada de Barcelona. [Tesis de máster]. Universidad de Barcelona, Barcelona, España. Disponible en http://hdl. handle.net/2445/56349

Padilla-Llano, S. E., Machado-Penso, M. V., Reyes-Schade, E., Larios-Giraldo, P. M., Cabrera-Sánchez, I., Martínez-Palacios, E., González-Forero, D. \& Tapias-Martínez, J. (2020). BARRIO EL PRADO un Museo Vivo para la Ciudad de Barranquilla. on thew@terfront. Public Art. Urban Design. Civic Participation. Urban Regeneration, 62(3), 3-46. https://doi.org/10.1344/waterfront2020.62.6.3

República de Colombia. Presidencia de la República. (26 de mayo de 2015). Decreto Único Reglamentario del Sector Vivienda, Ciudad y Territorio. [Decreto 1077]. Diario Oficial No. 49.523.

Rodríguez, G. (1983). Manual de diseño industrial. México, D.F.: GG.

Romero, O., Quintero, J., Cortes, O. y Botero, J. (2016). Código de Buenas Prácticas para el despliegue de redes de comunicaciones. Bogotá, D.C.: Comisión de Regulación de Comunicaciones, Ministerio de tecnologías de la información y las comunicaciones. Recuperado de https://www.crcom.gov.co/recursos_ user/2016/Informes/Codigo_Buenas_Practicas_2016.pdf
Rozadas, N. (2006). Contaminación Visual. Revista Académica del Equipo Federal de Trabajo, 15(35), [Revista en línea].

Sierra, A. y Guevara, O. (2016). Contaminación visual en centros históricos: un problema estético y de valor social de las ciudades actuales. Procesos Urbanos, 3, 133-142. https://doi. org/10.21892/2422085X.273

Vidal, T., Salas, X., Viegas, I., Esparza, D. y Padilla, S. (2012). El mural de la memoria y la Rambla Ciutat d'Asunción del barrio de Baró de Viver (Barcelona): repensado la participación ciudadana en el diseño urbano. Athenea Digital, 12(1), 29-53. Disponible en https://www.raco.cat/index.php/Athenea/article/view/250915

Western Australian Planning Comission. (1928). Statement of Planning Policy No. 5.2. [Telecommunications Infrastructure as ammended. [Online]. Available: https://www.joondalup.wa.gov.au/files/councilmeetings/2004/ Attach11brf161104.pdf

Emerson Martínez Palacios es Ma en Diseño Urbano, arte, ciudad y sociedad. PTC del departamento de Arquitectura y diseño de la Universidad de la Costa (Colombia). Director de la Maestría en Proyectos de Construcción Sostenible. Gestor de programas académicos y registro calificado. Centro de diseño e innovación CREATIVO. Director del grupo de investigación Ti. https://orcid. org/0000-0003-0472-9491 